\title{
Entropy driven phase transitions in colloid-polymer suspensions: Tests of depletion theories
}

\author{
S. Ramakrishnan \\ Departments of Chemical Engineering and Materials Research Laboratory, University of Illinois, \\ Urbana, Illinois 61801 \\ M. Fuchs ${ }^{\text {a) }}$ \\ Department of Physics and Astronomy, Edinburgh University, JCMB King's Building, Mayfield Road, \\ Edinburgh EH9 3JZ, United Kingdom \\ K. S. Schweizer \\ Departments of Chemical Engineering and Materials Science and Chemistry, University of Illinois, \\ Urbana, Illinois 61801 \\ C. F. Zukoski \\ Departments of Chemical Engineering and Materials Research Laboratory, University of Illinois, \\ Urbana, Illinois 61801
}

(Received 7 June 2001; accepted 17 October 2001)

\begin{abstract}
The phase behavior of model athermal silica (radius $R=50 \mathrm{~nm}$ )-polystyrene-toluene suspensions has been determined over nearly two orders of magnitude in polymer or colloid size asymmetry. Fluid-gel, fluid-crystal, and fluid-fluid transitions are observed as $R_{g}$, the polymer radius of gyration, increases. Based on the polymer concentration relative to the dilute-semidilute crossover density, $c_{p} / c_{p}^{*}$, as the relevant measure of depletion attraction, we find that suspension miscibility monotonically improves as $R_{g}$ increases for all colloid volume fractions. This trend is in contradiction to all classic depletion theories of which we are aware. However, the predictions of fluid-fluid spinodal phase separation by the microscopic polymer reference interaction site model integral equation theory of athermal polymer-colloid suspensions are in agreement with the experimental observations. Polymer-polymer interactions, chain fractal structure, and structural reorganizations are implicated as critical physical factors. A fluid-gel transition is observed in the one-phase region for $R_{g}=0.026 R$. The recently proposed dynamic mode-coupling theory is found to provide a nearly quantitative prediction of the gel line. With increasing $R_{g} / R$, gelation is predicted to require larger values of $c_{p} / c_{p}^{*}$ such that the nonergodicity transition shifts into the metastable region of the phase diagram in agreement with experiment. Comparison of the gelation behavior predicted based on the assumption that it is coincident with the static percolation line is also examined, with mixed results. (c) 2002 American Institute of Physics.
\end{abstract}

[DOI: $10.1063 / 1.1426413$ ]

\section{INTRODUCTION}

Mixtures of polymers and colloids are used to achieve desired material properties in paints, coatings, inks, and personal care products. Polymers are added to suspensions to stabilize the particles against aggregation, ${ }^{1,2}$ to induce aggregation, ${ }^{3,4}$ or as additives to alter the flow properties of the suspension. ${ }^{5,6}$ In many cases, polymers are added that do not adsorb to the particle surface and are thus free in solution. These free polymers can give rise to attractions between the particles due to excluded-volume effects. Polymer segments in the gap between two particles become increasingly restricted as the particle separation decreases and as a consequence lose translational and conformational entropy. This results in an exclusion of polymer segments from the interparticle gap and a reduction in the free energy of the ternary system. Models for this phenomenon are typically based on a

${ }^{a)}$ Permanent address: Physik-Department, Technische Universität München, 85747 Garching, Germany. pseudo-one-component description where the reduction in segment density between the particles creates an imbalance in osmotic pressure, resulting in a net particle attraction of entropic origin. In those approaches, the effect of the added polymer on the particles is described as a pair potential with the strength governed by polymer concentration through the polymer solution osmotic pressure $\Pi$ and a range characterized by the polymer radius of gyration $R_{g}$.

At a fixed particle density, as the nonadsorbing polymer concentration increases, suspensions often gel or phaseseparate. ${ }^{7-9}$ Control of these phase changes is important for achieving desired properties suited for particular applications. In this work we report a comprehensive study of the phase behavior of a model system of colloidal spheres of radius $R$ and a nonadsorbing polymer in which the size asymmetry ratio, $R_{g} / R$, is varied from much less than 1 to greater than 1 and as the polymer concentration is increased into the semidilute regime.

The first successful model to describe the effects of a

Konstanzer Online-Publikations-System (KOPS) 
single (dilute) nonadsorbing polymer on two-particle interactions was developed by Asakura and Oosawa. ${ }^{10}$ This model predicts that as two colloidal particles are brought to a center-to-center separation $r<2\left(R+R_{g}\right)$, an excluded volume develops in the gap between the particles into which the polymer cannot penetrate. The exclusion of the polymer from this gap gives rise to an imbalance of osmotic pressure that can be modeled as an effective attraction between the particles,

$U_{\mathrm{AO}}(r)=\left\{\begin{array}{l}\infty, \quad r<2 R \\ -\frac{4}{3} \pi d^{3} n_{p} k T\left[1-\frac{3 r}{4 d}+\frac{r^{3}}{16 d^{3}}\right], \quad 2 R<r<2 d \\ 0, \quad 2 d<r\end{array}\right.$

where $d=R+R_{g}$ characterizes the distance of closest approach of a polymer center of mass and a particle, $n_{p}$ is the polymer number density, and $k T$ is the thermal energy. In the Asakura-Oosawa (AO) model, the colloidal particles are represented by hard spheres [i.e., in the absence of a polymer, the particle pair potential is $u(r)=\infty$ for $r \leqslant 2 R$ and $u(r)=0$ for $r>\sigma]$. The polymers are treated as spherical objects that can pass freely through each other but interact with colloidal particles as hard spheres of radius $R_{g}$.

Building on the AO pair potential, Gast, Hall, and Russel $^{11}$ developed a hard-sphere perturbation theory to predict the phase behavior of colloid-polymer mixtures. Perturbing the hard-sphere solution structure with an attractive tail, this model predicts fluid-fluid and fluid-

transitions as functions of polymer and partir

tions and $R_{g} / R$. This theory qualitatively agrees with the experimental observations that for $R_{g} / R \geqslant 0.3$, thermodynamically stable fluid-fluid and fluid-crystal transitions will occur. For $R_{g} / R \leqslant 0.3$, the model predicts that the only stable phases are a fluid and a crystal in reasonable agreement with experiment. The thermodynamic perturbation approach is based on a pseudo-one-component model for suspension thermodynamics. As a consequence, the partitioning of polymer between the two fluid phases (or the fluid and crystalline phases) and three phase equilibria are not captured.

A generalization of this approach has been developed by Lekkerkerker et al. ${ }^{12}$ who construct a two-component meanfield theory based on an AO-like model to predict the phase behavior of colloid-polymer mixtures. This approach accounts for polymer partitioning at phase separation and is capable of predicting the existence of three phase regions for a range of polymer and colloid concentrations. The Lekkerkerker et al. theory qualitatively captures the observations of Ilett et al., ${ }^{13}$ who reported a range of polymer and particle concentrations where fluid and crystalline phases coexist. The key aspect of the theory is the change in solution free energy upon addition of polymer, which is determined by assuming that a void in the suspension must be created to insert a polymer sphere of volume $4 \pi R_{g}^{3} / 3$. This model is very similar to the approach taken by Asakura and Oosawa ${ }^{10}$ in the sense that colloids experience excluded-volume interactions with other colloids and polymers that are treated as spheres of radius $R_{g}$. Polymer-polymer interactions are ig- nored. The Gast, Hall, and Russel and Lekkerkerker et al. theories are fundamentally of the same nature and we refer to them collectively as "classical" approaches. The approximations by the classical theories are more appropriate when the polymer is in a $\theta$ solvent, the particles interact as hard spheres, the polymer is very dilute, and $R \gg R_{g}$.

As the polymer concentration increases towards and above the dilute-semidilute overlap concentration, $c_{p}^{*}$, the chains interpenetrate and polymer-polymer interactions become important. When $R_{g}>R$, particles can penetrate polymer coils, and treating the latter as hard spheres becomes an increasingly poor approximation. Ignoring these physical effects results in an overestimation of the polymer-induced depletion attraction in the classical approaches. As a consequence we anticipate these models will fail as $c_{p} / c_{p}^{*} \rightarrow 1$ and $R_{g} / R \rightarrow 1$, as pointed out in the original work of Gast, Hall, and Russel. ${ }^{11}$

Chatterjee and Schweizer ${ }^{14-16}$ recently developed an analytic polymer reference interaction site model (PRISM) integral equation theory based on the Percus-Yevick (PY) closure for describing polymer-induced depletion interactions between hard spherical particles with emphasis on calculating the second virial coefficient between the colloidal particles. PRISM is a two-component liquid-state approach ${ }^{17}$ that takes into account polymer structure and correlations on the segment scale and is, in principle, applicable for all $R_{g} / R$ ratios and polymer concentrations. In the dilute particle limit where polymer configurations are not altered by the presence of the particles, the PRISM-PY approach provides simple rking in this limit, Kulkarni et al. ${ }^{18,19}$ ractions between globular proteins in the presence of polyethylene glycol (PEG) through the measurement of the protein contribution to the suspension second virial coefficient $\left(B_{2}\right)$. For $R_{g} / R \ll 1$, the AO model predicts the observed dependence of $B_{2}$ on polymer concentration. However, as $R_{g} / R$ increases above unity, the AO model qualitatively fails, while the PRISM depletion potential accurately predicts subtle and qualitatively new changes in $B_{2}$ with $c_{p}, R_{g} / R$, temperature, and ionic strength. However, at nonzero colloid volume fractions the PRISM-PY theory does not predict athermal fluid-fluid phase separation in mixtures where polymers strongly repel (good solvent conditions). Fuchs and Schweizer ${ }^{20(a)}$ argue that this is due primarily to the neglect of nonlocal perturbations of polymer conformational entropy in the vicinity of hard particles, an effect well known in polymer physics. ${ }^{21}$ This observation motivated them to develop a qualitatively new closure approximation for polymer-colloid direct correlations called the modified Percus-Yevick (m-PY). The m-PY version of PRISM accounts for nonlocal conformational entropy effects in a predictive, thermodynamically self-consistent fashion.

Based on the m-PY PRISM theory, recent semianalytic calculations that employ a Gaussian thread description of polymer chains suggest that the inclusion of correlation effects is crucial for a successful description of the suspension thermodynamics and structure at high colloid densities. ${ }^{20(b)}$ Explicit consideration of the conformational entropic contributions of the polymers is required to address the packing of 
large polymers into the void space between colloidal spheres. The theoretical calculations of the static structure factor of colloids $^{20(a)}$ were in good agreement with the experimental data of Moussaid et al. ${ }^{22}$ in concentrated colloid-polymer mixtures. In its current state of development, the m-PY PRISM theory predicts entropy-driven fluid-fluid spinodal demixing, which appears to be in qualitative agreement with fragmentary experimental data on proteins, surfactant micelles, or inorganic and organic colloids mixed with polymers.

Our primary interest lies in understanding the phase behavior of colloid-polymer mixtures over a wide size ratio $\left(R_{g} / R\right)$ range under athermal, good solvent conditions where the polymer concentration increases into the semidilute regime. In addition, despite a considerable number of prior experimental and modeling studies, ${ }^{1-13}$ few quantitative comparisons of theoretical predictions and experiments have been made even when $c_{p} / c_{p}^{*} \ll 1$ and $R_{g} / R \ll 1$ where the $\mathrm{AO}$ and classical approaches are expected to be most accurate.

In this study we report quantitative comparisons between theoretical predictions and experimental determination of the phase behavior. We map out the phase diagram of a model system of hard-sphere colloids plus nonadsorbing polymerssterically stabilized colloidal silica and polystyrene (PS) in toluene, a good solvent for PS. The size ratio of polymer to colloid is varied over a wide range, $0.026 \leqslant R_{g} / R \leqslant 1.4$ and the influence of solvent quality is briefly addressed. The ability of standard models in the literature and PRISM-m-PY to predict the observed phase behavior is explored. While equilibrium models and experimental observations demonstrate that particles experiencing depletion interactions show fluid and crystalline phases at equilibrium, they also are known to produce gels. These gels are nonequilibrium structures associated with a loss of ergodic behavior and cannot be predicted from the equilibrium models discussed above. The location of the gel line and influence of the range of attraction on gelation are poorly understood. Recently, Bergenholtz and co-workers ${ }^{23}$ developed a dynamic mode-coupling theory for colloidal gelation. Here we discuss the relevance of this model to the phase behavior observed in suspensions of hard spheres containing nonadsorbing polymer in a good solvent.

\section{EXPERIMENTAL APPROACH}

Silica particles were synthesized through base-catalyzed hydrolysis and condensation in ethanol of tetra ethyl ortho silicate (TEOS) at $50{ }^{\circ} \mathrm{C}$ following the method of Stober, Fink, and Bohn. ${ }^{24}$ A seeded growth technique ${ }^{25}$ was then used to increase particle diameters and suspension weight fraction. Particles were sized using transmission electron microscopy (TEM) and dynamic light scattering yielding a particle diameter of $100 \pm 5 \mathrm{~nm}$. Particles were rendered hydrophobic using the method of van Helden, Jansen, and Vrij, ${ }^{26}$ which involves boiling of the silica-ethanol mixture with gradual addition of stearyl alcohol. The final boiling process was carried on for six hours at $210^{\circ} \mathrm{C}$, after which the reaction mixture was allowed to cool to room temperature. The

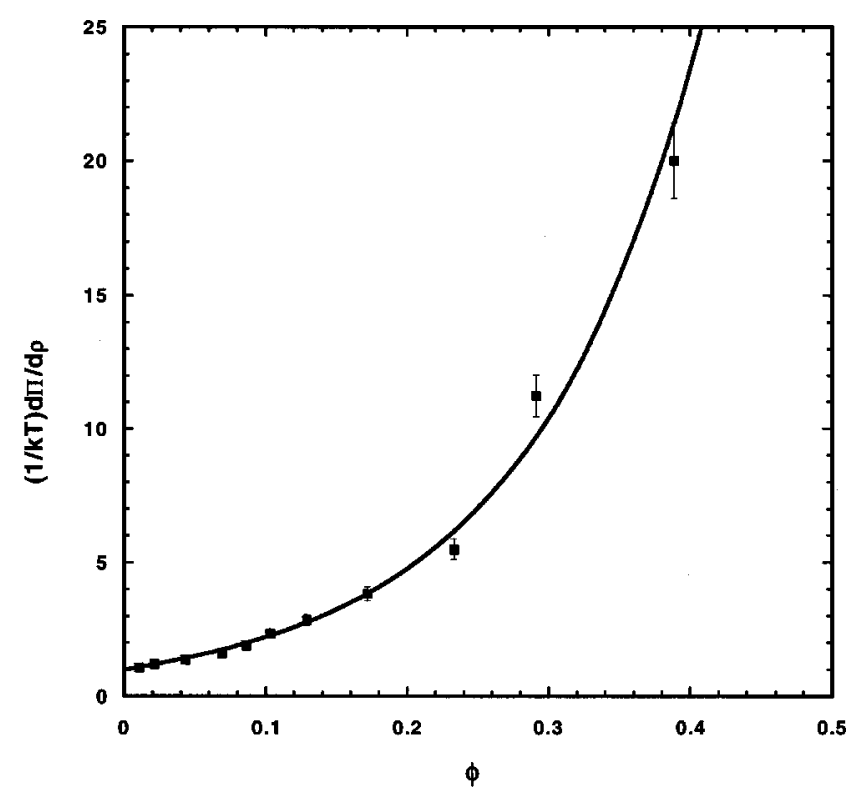

FIG. 1. Inverse dimensionless osmotic compressibility of silica colloids in toluene as a function of silica volume fraction. The solid line is the prediction from the Carnahan-Starling equation for hard spheres. Volume fraction was calculated from the mass fraction using the gravimetrically determined particle density $\rho_{c}=1.9 \mathrm{~g} / \mathrm{cm}^{3}$.

particles were washed by dissolving the solid mixture in chloroform followed by centrifugation and decantation. This process was repeated until there was no evidence of residual stearyl alcohol. The particles had a gravimetrically determined density of $\rho_{c}=1.9 \pm 0.04 \mathrm{~g} / \mathrm{cm}^{3}$. Suspensions were prepared by dispersing a known mass of dried silica powder in toluene followed by stirring to better disperse the powder. The volume fractions $\phi$ were also determined by dry weight using $\phi=c / \rho_{c}$, where $c$ is the mass concentration (mass/ volume) of the silica in suspension. In the absence of polymer, sedimentation was not observed for any of the volume fractions used in this work over the time period in which the experiments were done.

Polystyrenes of five different molecular weights, 1.88 $\times 10^{6}, 5.5 \times 10^{5}, 2.124 \times 10^{5}, 2.93 \times 10^{4}$, and $2.43 \times 10^{3}$ were purchased from Aldrich Chemical Company. The ratio of the weight average to number average molecular weight is 1.03 , implying very monodisperse samples. Polymer solution properties were characterized using static light scattering.

Phase diagram experiments were done in a 4-ml Fisherbrand disposable culture tubes. The polymer solution of a given concentration was slowly added to a silica suspension of a given volume fraction until a phase separation or gelation was visually observed.

\section{RESULTS}

\section{A. Hard spheres}

Silica spheres coated with octadecanol and suspended in toluene behave as hard spheres above $\sim 25^{\circ} \mathrm{C}$. We chose to work at $35^{\circ} \mathrm{C}$ based on measurement of the suspension osmotic compressibility at different temperatures. For $T$ $<30{ }^{\circ} \mathrm{C}$, the compressibility showed indications of attractions. Shown in Fig. 1 is a plot of the dimensionless inverse 
TABLE I. Radius of gyration $\left(R_{g}\right)$ and polymer overlap concentration $\left(c_{p}^{*}\right)$ for the five different polymers used in this work. The size ratio $R_{g} / R$ is also given for each polymer used $(R=50 \mathrm{~nm})$.

\begin{tabular}{lccc}
\hline \hline $\begin{array}{c}\text { Molecular } \\
\text { weight }(\mathrm{g} / \mathrm{mol})\end{array}$ & $R_{g}(\mathrm{~nm})$ & $R_{g} / R$ & $c_{p}^{*}(\mathrm{mg} / \mathrm{ml})$ \\
\hline $2.43 \times 10^{3}$ & 1.29 & 0.026 & 418.79 \\
$2.93 \times 10^{4}$ & 5.74 & 0.115 & 57.14 \\
$2.124 \times 10^{5}$ & 18.85 & 0.377 & 11.72 \\
$5.5 \times 10^{5}$ & 33.37 & 0.667 & 5.47 \\
$1.88 \times 10^{6}$ & 69.77 & 1.395 & 2.05 \\
\hline \hline
\end{tabular}

osmotic compressibility $(1 / k T)(d \Pi / d \rho)$ as a function of volume fraction $\phi$ for silica in toluene at $35^{\circ} \mathrm{C}$. These measurements were made using the turbidity method of Horner, Topper, and Ballauf. ${ }^{27}$ Comparison of measured values and those calculated with the Carnahan-Starling equation of state $^{28}$ for hard spheres requires converting mass concentration to volume fraction. Often the adjustable parameter used to achieve this agreement is $\rho_{c}$. In our case, the comparison is made with the gravimetrically determined value of 1.9 $\mathrm{g} / \mathrm{cm}^{3}$. The agreement with no adjustable parameters is strong evidence that the particles in the absence of polymer interact as hard spheres.

\section{B. Polymer characterization}

Static light scattering was used to characterize the 1.88 $\times 10^{6} M_{w}$ polymer in toluene. The radius of gyration $\left(R_{g}\right)$ and the second virial coefficient $\left(B_{2}\right)$ were in good agreement with literature values. The good-solvent scaling law $\left(R_{g} \propto M_{w}^{3 / 5}\right)$, confirmed by Berry, ${ }^{29}$ was then used to calculate the radii of gyration for the other polymers. The values are shown in Table I. The polymer overlap concentration $c_{p}^{*}$ was estimated from the standard equation

$$
c_{p}^{*}=\frac{3 M_{w}}{4 \pi R_{g}^{3} N_{A}},
$$

where $M_{w}$ is the molecular weight of the polymer and $N_{A}$ is the Avogardo number. The $c_{p}^{*}$ values are also tabulated in Table I.

\section{Phase diagram}

The location of phase boundaries were determined by starting with a known volume of dense suspension of particles with $c_{p}=0$ (no polymer) and diluting this suspension with a known volume of concentrated polymer solution with $\phi=0$. The dilutions were carried out in small steps $(20 \mu \mathrm{l})$ and after mixing the solutions were allowed to sit from five minutes to several days to allow a phase separation to occur. The phase diagram of silica suspensions for five different molecular weight polystyrenes in toluene is shown in Fig. 2. Data points represent the values of $c_{p}$, which is the average of the highest observed polymer concentration when the suspension is homogenous and the polymer concentration when phase separation first occurs. Estimates of uncertainty of the phase boundary are determined as corresponding to $\pm 10 \mu \mathrm{l}$. The value of $R_{g} / R$ varies from 0.026 to 1.395 as the polystyrene molecular weight varies from $2.43 \times 10^{3}$ to 1.88

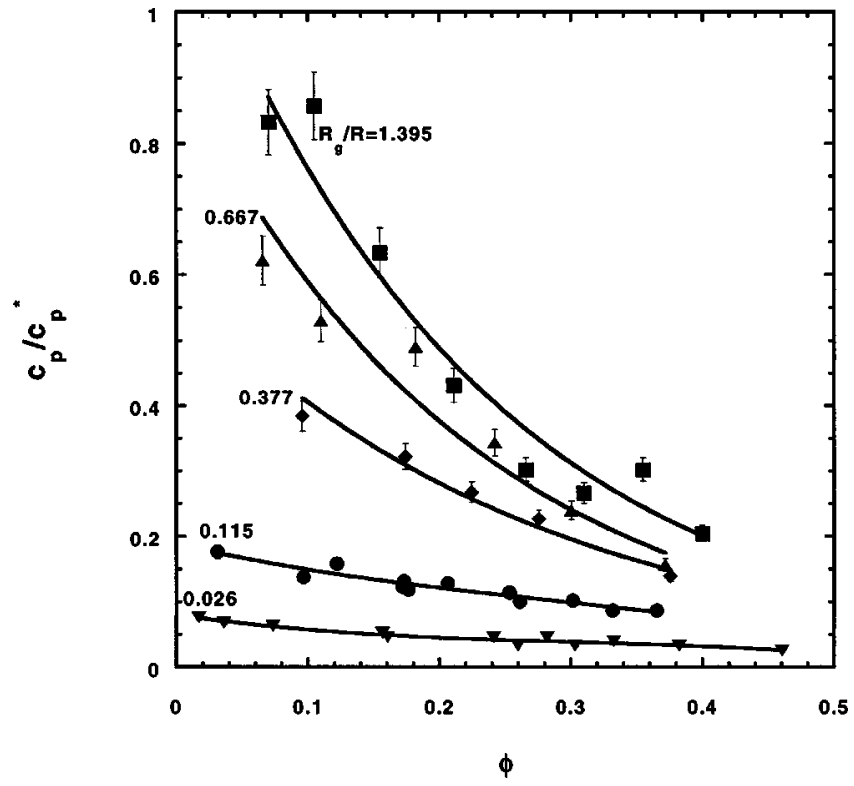

FIG. 2. Experimental phase diagrams of silica in toluene in the presence of polystyrene for five different values of $R_{g} / R$. The horizontal axis is the colloid volume fraction $\phi$ and the vertical axis is the dimensionless polymer concentration $\left(c_{p} / c_{p}^{*}\right)$. The curves are drawn to guide the eye.

$\times 10^{6} \mathrm{~g} / \mathrm{mol}$. The phase diagrams are plotted in terms of a dimensionless polymer concentration $c_{p} / c_{p}^{*}=c_{\text {poly }} 4 \pi / 3 R_{g}^{3}$ (where $c_{\text {poly }}$ is the polymer chain number density) consistent with theoretical approaches in the dilute and semidilute polymer concentration regimes. The curves are drawn to guide the eye. Below the curves there is a single-phase fluid region, while with increasing colloid or polymer concentration phase separation occurs.

When $R_{g} / R$ is 0.026 the points indicate the formation of a "gel." These gels are space-filling amorphous, solidlike suspensions. For $\phi<0.35$, these gels are weak and over a period of several hours settle under their own weight, producing a dense amorphous compact and a clear (particlefree) solution. At higher volume fractions, these gels are stronger and do not settle and densify. Along the indicated boundary, there was an abrupt transition from a transparent low-viscosity suspension to a very turbid, high-viscosity (or solidlike) suspension.

When $R_{g} / R$ is 0.115 the suspension separates into a dense lower phase and a dilute upper phase. We suspect this lower phase to consist of crystals but this has not yet been confirmed with $x$-ray scattering. The dense phase had mechanical relaxation times of several hours, suggesting the presence of a yield stress. There are several differences between the phase boundaries in the $R_{g} / R=0.026$ and 0.115 cases: (1) For $R_{g} / R=0.026$, the gel formation was immediate and on settling the supernatant was particle free. (2) For $R_{g} / R=0.115$, the phase transition occurred slowly and there was a coexistence between dense solidlike and dilute phases. (3) Sedimentation of the dense phase was delayed for $R_{g} / R=0.026$, while for $R_{g} / R=0.115$, the sedimentation occurred more rapidly.

For the three higher molecular weights the phase boundary is clearly a liquid-liquid phase separation. In such 
samples, a meniscus separating two fluid phases appears a few minutes after mixing. The bottom phase is more concentrated in colloid than the upper phase, and both of the phases flow easily. Our results qualitatively agree with the observations of Ilett et al., ${ }^{13}$ who mapped out the phase diagram of colloid-polymer mixtures for three different size ratios of $0.08,0.24$, and 0.57 . A central result of Fig. 2 is that the homogenous fluid-mixture phase is monotonically stabilized as $R_{g}$ increases.

Figure 3(a) shows the effect of solvent quality of the polymer solution on the location of the phase boundary. In both toluene and cyclohexane, particles interact as hard spheres. However, toluene is a good solvent for polystyrene while at $30^{\circ} \mathrm{C}$ cyclohexane is nearly a $\theta$ or ideal solvent. The polystyrene radius of gyration decreases from $69.7 \mathrm{~nm}$ in a good solvent to $42 \mathrm{~nm}$ in cyclohexane at $35^{\circ} \mathrm{C}$. A unique and unambiguous comparison of good- and $\theta$-solvent behavior is subtle since both intracoil and interaction properties depend on solvent quality. Normalizing the polymer concentration with $c_{p}^{*}$ is one way comparison of the effects of different solvent conditions on the effective polymer volume fraction can be made. From Fig. 3(a) we see that at fixed $\phi$, a lower value of $c_{p} / c_{p}^{*}$ is required to induce phase separation as solvent quality is reduced. Since reduction of solvent causes a decrease of $R_{g} / R$, this trend is qualitatively consistent with the good-solvent results of Fig. 2. In addition, the reduction of polymer-polymer repulsions in the $\theta$ solvent is presumably another source of reduced polymer-colloid miscibility. However, we note that in terms of absolute concentration $c_{p}$, phase separation in the $\theta$ solvent requires more polymer since $c_{p}^{*}$ is significantly larger in the $\theta$ solvent. Interestingly, comparison of the $\theta$-solvent boundary with the $R_{g} \cong 33 \mathrm{~nm}$ ( $\left.R_{g} / R=0.667\right)$ good-solvent binodal in Fig. 2 suggests that even at fixed $R_{g}$ the phase separation occurs at lower values of $c_{p} / c_{p}^{*}$ under ideal solution conditions.

The effects of variable solvent quality are seen again in Fig. 3(b), where we compare the value of $c_{p} / c_{p}^{*}$ required to induce a phase separation at fixed $\phi$ as a function of $R_{g} / R$. Included in this figure are data from Ilett et al., ${ }^{13}$ where phase boundaries are for PMMA hard-sphere colloids $(R$ $\cong 200 \mathrm{~nm}$ ) plus polystyrene in a near- $\theta$ solvent. For $R_{g} / R$ $\geqslant 0.1$ samples, we again see that a smaller value of $c_{p} / c_{p}^{*}$ is required to induce a phase separation in a near- $\theta$ solvent than in a good solvent. Of equal importance in Fig. 3(b) is that for solvents of both qualities, the value of $c_{p} / c_{p}^{*}$ required to induce phase separation is a monotonically increasing function of $R_{g} / R$. Note that for $R_{g} / R \geqslant 0.25$, the experimental points of Ilett et al. ${ }^{13}$ show fluid-fluid demixing, whereas for smaller size ratios crystallization occurs first.

\section{COMPARISONS WITH THEORY}

\section{A. Classic free-energy approaches}

The theory of Lekkerkerker et al. ${ }^{12}$ for the Helmholtz free energy of a system of $N_{c}$ colloidal particles and $N_{p}$ polymer molecules in a volume $V$ is written

$$
F=F_{c}\left(N_{c}, V\right)+F_{p}\left(N_{p}, \alpha V\right),
$$

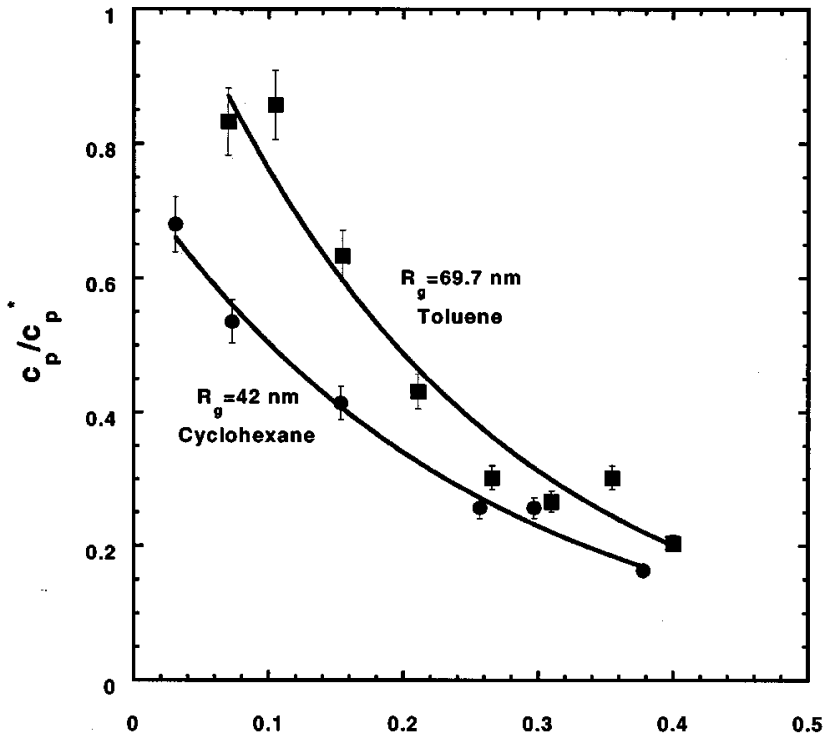

(a)

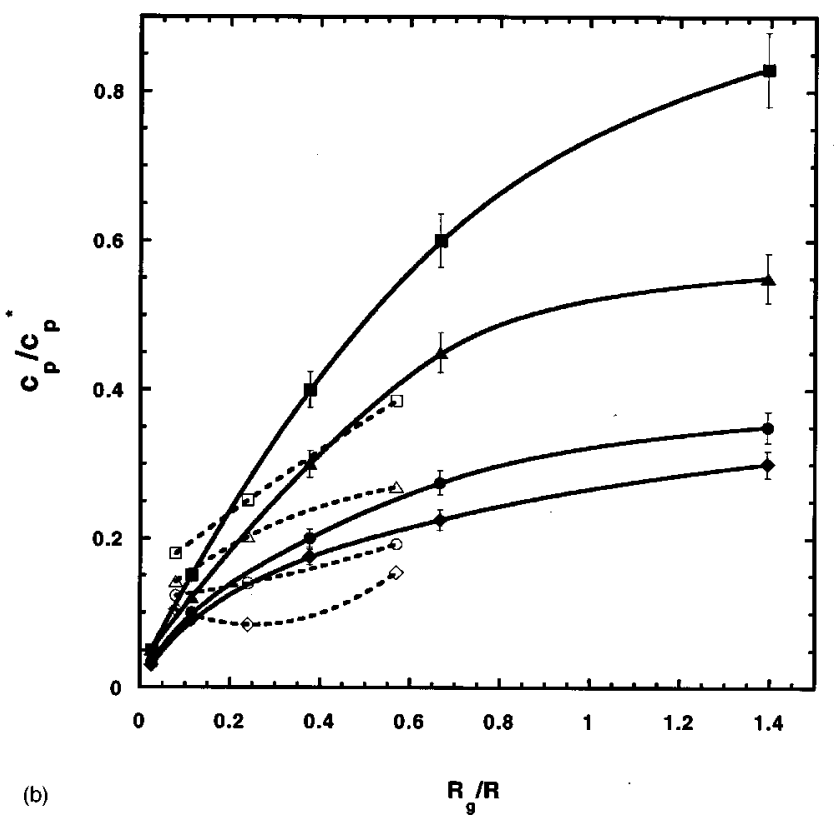

FIG. 3. (a) Phase diagram of silica in toluene (squares) and cyclohexane (circles) in the presence of $1.88 \times 10^{6}$ molecular weight polystyrene. The curves are drawn to guide the eye. The size ratio $R_{g} / R$ varies from 1.395 in toluene to 0.84 in cyclohexane. (b) Reduced polymer concentration required for phase separation at a fixed colloid volume fraction $\phi$ as a function of $R_{g} / R$ for the experimental system used in this work and that of Ilett $e t$ al. (Ref. 13). The solid symbols are the experimental points for $\phi=0.1$ (squares), 0.2 (triangles), 0.3 (circles), and 0.35 (diamonds), respectively. The open symbols are results from Ilett et al. (Ref. 13). The curves are drawn to guide the eye.

where $F_{c}$ and $F_{p}$ are the colloid and polymer contribution to the free energy, respectively. $F_{p}$ is coupled to the colloid concentration solely via the chemical potential or free energy for inserting a single polymer in the solution volume not occupied by the particles, $V_{\text {free }}=\alpha V$. Employing the wellknown scaled particle expression, or equivalently the Percus-Yevick result, for the chemical potentials of a mixture of hard spheres, ${ }^{12,30}$

$$
\alpha=(1-\phi) \exp \left[-A \gamma-B \gamma^{2}-C \gamma^{3}\right],
$$




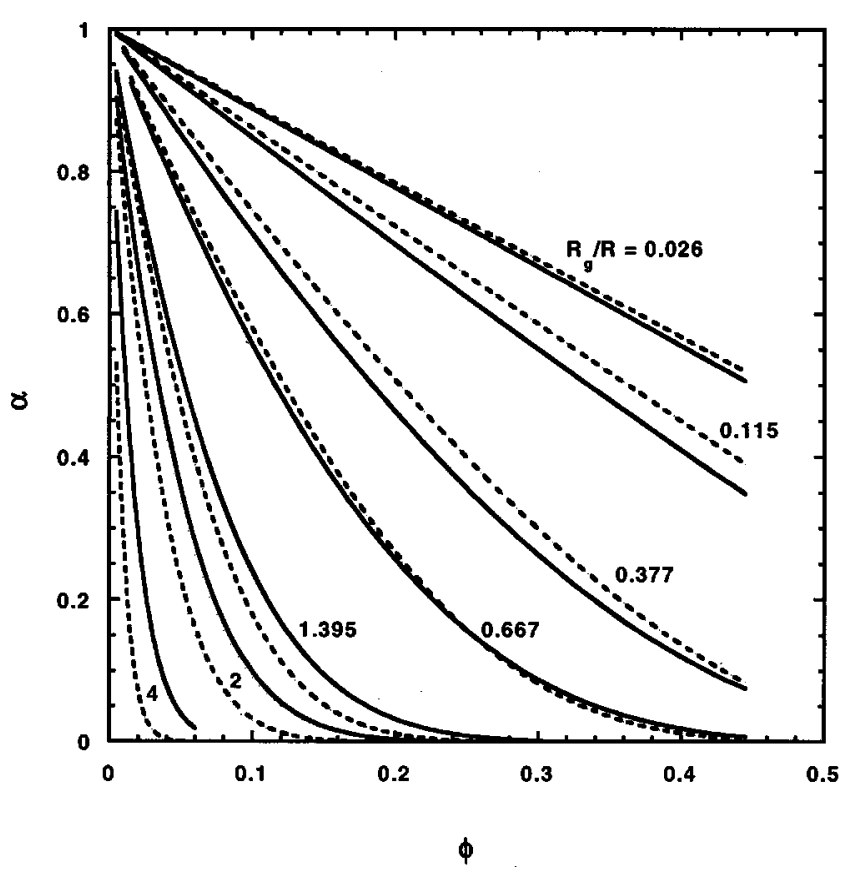

FIG. 4. Free-volume fraction parameter as a function of colloid volume fraction $\phi$ for different values of $R_{g} / R$. The solid lines are the m-PY PRISM results [Eqs. (5) and (6)] and the dashed lines correspond to Eq. (4).

where $\gamma=\phi /(1-\phi), A=3 \xi+3 \xi^{2}+\xi^{3}, B=9 \xi^{2} / 2+3 \xi^{3}, C$ $=3 \xi^{3}$, and $\xi=R_{g} / R$. The same expression for $\alpha$ is used for both the fluid and solid phases. This approach assumes the polymers are hard spheres and thus a spherical hole of radius $R_{g}$ must be created in the suspension before the polymer can be inserted.

In the m-PY PRISM approach, ${ }^{20}$ polymers are realistically modeled as connected chains of monomer units. The free-volume fraction, $\alpha_{\mathrm{m}-\mathrm{PY}}$, is given by ${ }^{20(\mathrm{~b})}$

$$
\alpha_{\mathrm{m}-\mathrm{PY}}=(1-\phi) \exp [-Q],
$$

where

$$
\begin{aligned}
Q= & \frac{6 \phi \varpi\left(1+4 / \lambda_{1}\right)}{1-\phi}+\frac{6 \phi \varpi^{2}(2+\phi)\left(1+2 / \lambda_{1}\right)}{(1-\phi)^{2}} \\
& +\frac{6 \varpi \phi\left(4 / \lambda_{1}^{2}-2 / \lambda_{1}\right)}{\left(1+\lambda_{1} \varpi\right)\left[1+\lambda_{1} \varpi-\phi\left(1-2 \lambda_{1} \varpi\right)\right]}
\end{aligned}
$$

and $\lambda_{1}=1+\sqrt{5}$ and $\varpi=R_{g} /(2 \sqrt{2} R)$. Figure 4 compares the two $\alpha$ 's for the values of $R_{g} / R$ used in this work. The differences are small for the present $R_{g} / R$ values. However, when $R_{g} / R \gg 1$, differences between the two approaches become large ${ }^{20(\mathrm{~b})}$ with the Lekkerkerker et al. approach severely under predicting $\alpha$. Note that use of Eq. (4) or (6) does not account for the work required to insert the polymer into a nonzero polymer concentration solution.

In the classical approaches, polymer molecules are assumed to be noninteracting ("ideal"), yielding

$$
F_{p}\left(N_{p}, \alpha V\right)=k T n_{p} V \log \left(n_{p} / \alpha\right),
$$

where $n_{p}=N_{p} / V$. The colloid contribution to the free energy is given by
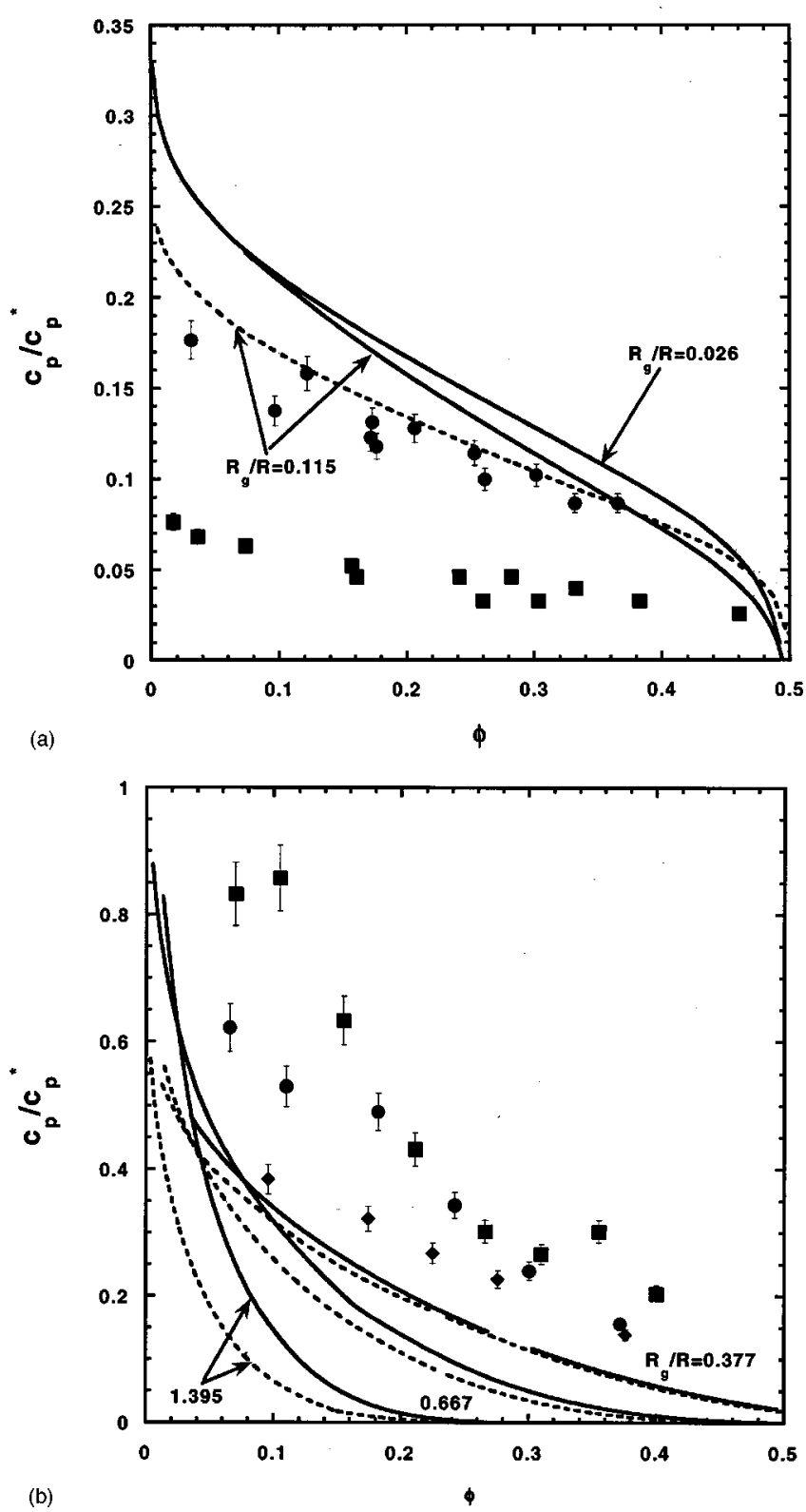

FIG. 5. Experimental phase diagrams of silica in toluene in the presence of polystyrene for different values of $R_{g} / R$ compared with model calculations. The solid symbols are the experimental points. The solid (dashed) curves are from the Lekkerkerker et al. (Gast, Hall, and Russel) theory. (a) Results for $R_{g} / R$ of 0.026 (squares) and 0.115 (circles). (b) Results for $R_{g} / R$ of 0.377 (diamonds), 0.667 (circles), and 1.395 (squares).

$$
F_{c}\left(N_{c}, V\right)=\frac{3 k T}{4 \pi R^{3}} V \phi \int \frac{Z}{\phi} d \phi,
$$

where $Z$ is the hard-sphere compressibility $(Z=\Pi / \rho k T$, where $\Pi$ is the osmotic pressure and $\rho$ is the number of particles per unit volume). The phase diagram is calculated by enforcing the standard equilibrium conditions. ${ }^{12}$ In the fluid phase $Z$ is determined from the Carnahan-Starling equation of state, while for the crystal phase the Hall equation of state ${ }^{31}$ for hard spheres is used.

Shown in Fig. 5(a) are comparisons of Lekkerkerker et al. theory ${ }^{12}$ with experimental data for $R_{g} / R=0.026$ and 0.115 . When $R_{g} / R<0.3$, there is no stable fluid-fluid phase boundary. As a consequence the curves shown in Fig. 5(a) 
are for the fluid-crystal boundary. Experimentally a gel is observed when $R_{g} / R=0.026$. For $R_{g} / R=0.115$, the theory is qualitatively correct but quantitatively underpredicts the consequences of depletion attraction.

As the molecular weight of polystyrene is increased, the polymer size becomes comparable to that of the colloid. Shown in Fig. 5(b) are comparisons of the Lekkerkerker et al. theory with experimental data for $R_{g} / R=0.377,0.667$, and 1.395, where the equilibrium fluid-fluid phase transitions are predicted and observed. By replacing $n_{p}$ in Eq. (1) by $\alpha n_{p}$, the first-order perturbation theory of Gast, Hall, and Russe $^{11}$ can be recast to account for changes in the strength of the depletion attraction with particle volume fraction. This model then predicts phase transitions that are very similar to those of the Lekkerkerker et al. theory [as shown in Figs. 5(a) and 5(b)].

Of particular significance are the qualitatively incorrect predictions of the classical theories for the dependence of $c_{p} / c_{p}^{*}$ at the phase boundary on $R_{g} / R$. As discussed above, and shown in Fig. $5, c_{p} / c_{p}^{*}$ is observed experimentally to increase with $R_{g} / R$ while the classic theories predict the opposite trend. The deviations between theory and experiment become increasingly pronounced as $R_{g} / R$ or $\phi$ increase.

The comparisons in Fig. 4 show that the origin of the failure of classic approaches is not associated with the $c_{p}$ $\rightarrow 0$ single-polymer insertion chemical potential. Hence, other physical considerations must be examined. (1) Polymer-polymer repulsions at nonzero $c_{p}$ : In good solvents, such effects would act to reduce an AO-like depletion attraction, and above $c_{p}^{*}$ the physical mesh of the polymer solution must be accounted for. In addition, colloids mediate an effective attraction between interpenetrating polymer coils. $^{20(b)}$ Since the second virial coefficient of polymer molecules $B_{2, p p} \propto R_{g}^{3}$, versus the second virial coefficient of the colloidal particles $B_{2, c c}\left(c_{p} \rightarrow 0\right) \propto R^{3}$, polymer-polymer correlations and colloid-induced clustering are expected to become increasingly relevant as $R_{g} / R$ increases. As an indirect consequence, polymer-colloid repulsions will be modified from their $c_{p} \rightarrow 0$ behavior. (2) Many-body depletion effects (e.g., the effect of a third particle on the interaction of a pair due to alteration in polymer configuration produced by the third particle) beyond the simple effective one-component description based on the pair-decomposable (AO) attractions. (3) Failures of thermodynamic perturbation (hightemperature) theory: At nonzero polymer concentrations, polymers will mediate local and long-distance changes in colloid structure and free volume that will depend on $R_{g} / R$, $\phi$, and $c_{p} / c_{p}^{*}$.

\section{B. Adhesive hard-sphere and square-well equation of state models}

To address consideration (3) above within a pseudo-onecomponent simple-fluid approach we consider two nonperturbative approaches commonly employed in colloid science for attractive-particle fluids. The first is Baxter's adhesive hard-sphere (AHS) model. The fluid-fluid spinodal ${ }^{32,33}$ is computed from Baxter's exact solution of the Percus-Yevick integral equation: $\frac{1+2 \phi}{6(1-\phi)}-\tau_{s}-\frac{\phi}{1-\phi}+\left[\left(\tau_{s}+\frac{\phi}{1-\phi}\right)^{2}-\frac{\phi(\phi+2)}{6(1-\phi)^{2}}\right]^{1 / 2}=0$

with the condition

$$
\left(\tau_{s}+\frac{\phi}{1-\phi}\right)^{2}-\frac{\phi(\phi+2)}{6(1-\phi)^{2}} \geqslant 0 .
$$

Here $\tau_{s}$ is the stickiness parameter defining the integrated strength of attraction. Following standard procedure, if Eq. (10) is not satisfied, then the spinodal is calculated by setting the equation equal to zero. The AHS spinodal is then mapped on to the colloid-polymer phase diagram by relating $\tau_{s}$ and $c_{p} / c_{p}^{*}$ in two different ways. In both methods, the first step is to fix the size ratio $R_{g} / R$ and then to calculate the polymer concentration required for phase separation, $c_{p} / c_{p}^{*}$.

The first technique ( $B_{2, c c}$ mapping) equates the second virial coefficient of the AHS $\left(B_{2, c c}^{\mathrm{AHS}}\right)$ and AO $\left(B_{2, c c}^{\mathrm{AO}}\right)$ potentials to calculate $c_{p} / c_{p}^{*}$ as a function of $\tau_{s} . B_{2, c c}^{\mathrm{AHS}}$ and $B_{2, c c}^{\mathrm{AO}}$ are given by the following equations:

$$
\begin{aligned}
& \frac{B_{2, c c}^{\mathrm{AHS}}}{B_{2, c c}^{\mathrm{HS}}}=1-\frac{1}{4 \tau_{s}}, \\
& \frac{B_{2, c c}^{\mathrm{AO}}}{B_{2, c c}^{\mathrm{HS}}}=1+\frac{3}{8} \int_{2}^{2\left(1+R_{g} / R\right)}\left(1-e^{-U_{\mathrm{AO}}(s)}\right) s^{2} d s,
\end{aligned}
$$

where $B_{2, c c}^{\mathrm{HS}}\left(=16 \pi / 3 R^{3}\right)$ is the hard-sphere second virial coefficient, and $U_{\mathrm{AO}}$ is the $\mathrm{AO}$ interaction potential [Eq. (1)] with the $n_{p} \rightarrow \alpha n_{p}$ free-volume correction. $\tau_{s}$ is chosen such that $B_{2, c c}^{\mathrm{AHS}}=B_{2, c c}^{\mathrm{AO}}$.

In the second technique $[U(r)$ potential mapping] the AHS spinodal was first mapped onto a square-well potential. This was done by equating the second virial coefficients of the AHS and the square-well models,

$$
1-\frac{1}{4 \tau_{s}}=1+[\exp (\beta \epsilon)-1]\left[\left(1+\frac{\Delta}{\sigma}\right)^{3}-1\right],
$$

where $\beta \epsilon>0$ is the strength of the square-well attraction and $\Delta / \sigma$ the dimensionless spatial range. The AHS model is meant to mimic colloids that experience very-short-range attractions, $\Delta / \sigma \ll 1$. Thus, for simplicity we choose to perform the mapping in this limit. Equating the square well $\beta \epsilon$ with its AO analog from Eq. (1) and requiring the (threedimensional) integrated strength of the $\mathrm{AO}$ and square well attractions are equal yield

$$
\begin{aligned}
& \frac{\Delta}{\sigma}=\frac{1}{3} \frac{R_{g}}{R}, \\
& \beta \epsilon=\frac{3}{2} \frac{R}{R_{g}}\left(\frac{c_{p}}{c_{p}^{*}}\right) \alpha^{-1},
\end{aligned}
$$

where $\alpha$ is the polymer free-volume fraction.

The spinodal curves for both mappings are shown in Fig. 6. Values of $\alpha$ computed by the accurate PRISM/m-PY approach are used. As can be seen from Fig. 6, the results of both mappings are similar. However, both are in poor agreement with experiment. The correct ordering of the fluid-fluid phase boundaries with increasing $R_{g} / R$ is only found in the $\phi \rightarrow 0$ limit where only second virial coefficient information 


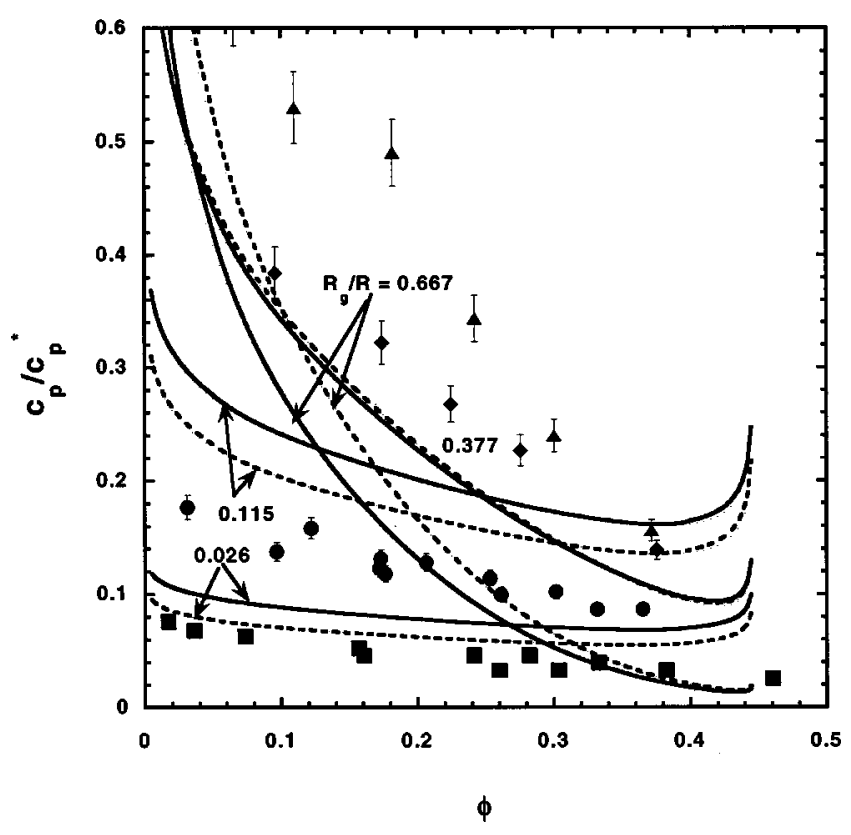

FIG. 6. AHS model spinodal curves based on the PY theory obtained by two mappings onto the colloid-polymer problem for four different values of $R_{g} / R$. The solid symbols are the experimental points: $R_{g} / R=0.026$ (squares), 0.115 (circles), 0.377 (diamonds), and 0.667 (triangles). The solid curves employ the $B_{2, c c}$ mapping and the dashed curves use the $U(r)$ potential mapping.

enters. Agreement with experiment clearly worsens as $R_{g} / R$ and/or $\phi$ increases, and the multiple "curve crossings" demonstrate that the AHS model has not solved the qualitative problem the classical theories display.

As an attempt to use an even more accurate onecomponent equation of state, we compute phase diagrams based directly on computer simulations of square-well fluids plus the same two mappings onto the polymer-colloid problem discussed above. In order to calculate the phase diagrams, the empirical fit formula developed by Ramakrishnan and Zukoski, ${ }^{34}$ which captures well the computer-simulated equation of state for square well fluids, is used:

$$
\frac{\Pi}{\rho k T}=1+\frac{B_{2}^{\mathrm{HS}} \rho}{\left(1-\rho / \rho_{0}\right)^{2}}+\frac{\rho a(\epsilon, \Delta)}{\left[1-\rho / \rho_{b}(\Delta)\right]^{3}},
$$

where $\Pi$ is the osmotic pressure, $\rho$ is the particle number density, $\rho_{0}=1.605 \sigma^{-3}$, and $a(\epsilon, \Delta)$ is a function of well depth. Equation (16) provides an excellent approximation for the machine calculations of square wells with $a(\epsilon, \Delta)$ and $\rho_{b}(\Delta)$ chosen to match values of $\epsilon / k T$ and $\rho \sigma^{3}$ at the critical point of the fluid-fluid phase transition. Values are given in Table II for selected values of $\Delta$. We find this correlation yields $\Pi / \rho k T$ to within $10 \%$ of the machine calculations reported by Heyes and Aston ${ }^{35}$ and predicts critical temperatures and densities to within 10\%. Due to the lack of analytical results for the solid state of square well and AHS solids, we follow Asherie, Lomakin, and Benedek ${ }^{36}$ in approximating the chemical potential of the solid phase as

$$
\mu_{s}=\mu_{0}-n_{s}(\epsilon / 2 k T)-3 \ln (\Delta),
$$

where $n_{s}$ is the number of nearest neighbors in the solid phase and $\mu_{0}$ is a constant that is the same in both the fluid
TABLE II. Parameters used in the square well equation of state [Eq. (16)] as calculated from the fluid-fluid critical point. Note that $a(\epsilon, \Delta)$ in Eq. (16) is defined as $a(\epsilon, \Delta)=(\epsilon / k T) f(\Delta)$. The strength of attraction $(\epsilon / k T)_{c}$ and the volume fraction $\phi_{c}$ at the critical point are also tabulated.

\begin{tabular}{lllcc}
\hline \hline \multicolumn{1}{c}{$\Delta$} & $f(\Delta)$ & $\rho_{b}(\Delta) \sigma^{3}$ & $(\epsilon / k T)_{c}$ & $\phi_{c}$ \\
\hline 0.004 & -0.554 & 2.563 & 5.122 & 0.266 \\
0.015 & -0.768 & 2.585 & 3.725 & 0.263 \\
0.1 & -1.558 & 2.83 & 2.001 & 0.239 \\
0.25 & -2.8 & 3.5 & 1.287 & 0.205 \\
\hline \hline
\end{tabular}

and solid phases. This model assumes the solid phase is incompressible. As a consequence, locating the phase boundary does not require equating pressures of the coexisting phases. In the square-well equation of state, the gas-liquid critical point becomes metastable with respect to fluidcrystal equilibrium when $\Delta=0.35$ and $\epsilon / k T=1.04$. Based on our $B_{2}$ mapping of the square well model onto the AO model, this corresponds to an $R_{g} / R$ value of 1.078.

The results are shown in Fig. 7, where the experimental polymer concentration $c_{p} / c_{p}^{*}$ calculated using $\alpha_{\mathrm{m}-\mathrm{PY}}$ is plotted as a function of $\phi$. For $R_{g} / R=0.026$ and 0.115 , the fluid part of the fluid-solid phase transition is shown, while for $R_{g} / R=0.377$ and 0.667 the fluid part of gas-fluid phase transition is depicted. Better agreement with experiment is found than for the AHS model, but it is still very poor. Note that the close agreement of the theoretical curve and the experimental gelation data for $R_{g} / R=0.026$ does not imply the theory is accurate. Thus, it is clear from both Figs. 6 and 7 that the idea of replacing the polymer-colloid mixture with a one-component particle fluid interacting via an effective pair

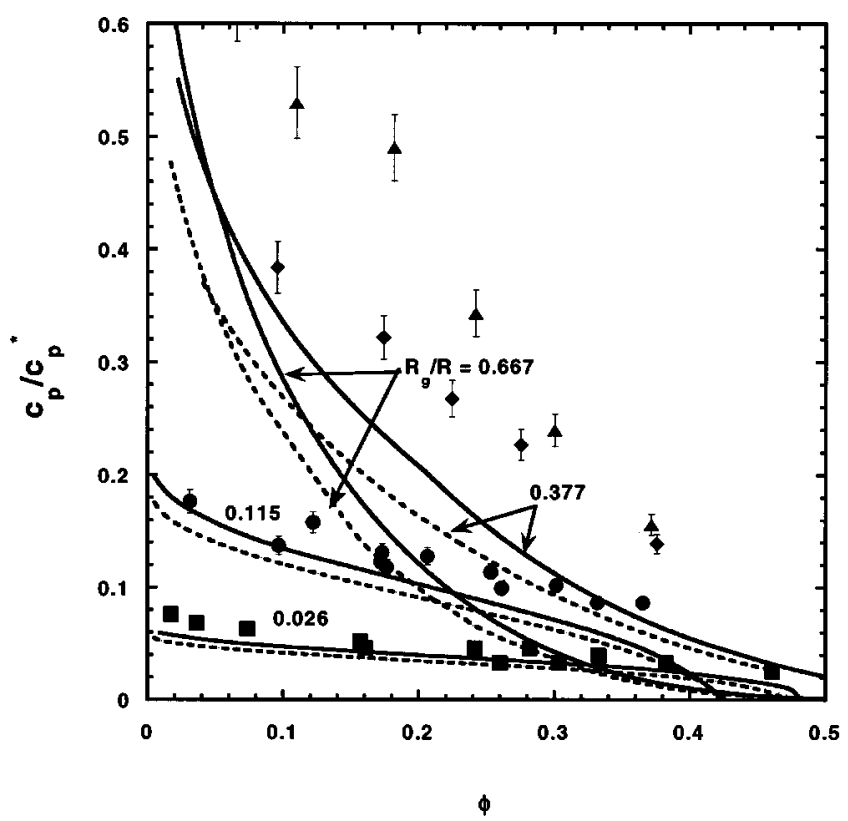

FIG. 7. Phase diagrams computed from the square-well equation of state using two mappings onto the colloid-polymer phase diagram and compared with the experimental data. The solid symbols are the experimental points for $R_{g} / R=0.026$ (squares), 0.115 (circles), 0.377 (diamonds), and 0.667 (triangles), respectively. The solid curves are calculations from the $B_{2, c c}$ mapping and the dashed curves are calculations from the $U(r)$ potential mapping. 
interaction potential does not capture the behavior of the phase boundaries. Phase transition lines predicted for different values of $R_{g} / R$ cross each other as $\phi$ increases, while the experimental phase boundaries shift up monotonically with increasing $R_{g} / R$ and do not cross each other. Again, only at low $\phi(\phi<0.1)$ is there a systematic trend in the calculated phase diagrams as $R_{g} / R$ increases, which matches the experiments. Thus, we believe it is essential to confront points (1) and (2) raised at the end of Sec. IV A in order to understand the experimental observations.

\section{PRISM theory}

PRISM theory has been recently generalized to treat athermal (good-solvent) polymer-colloid suspensions based on a new thermodynamically self-consistent treatment of polymer-particle direct correlations. ${ }^{20}$ This theory accounts for all three of the physical effects enumerated at the end of Sec. IV A, albeit within an approximate integral equation framework. Physically, by accounting for these effects, one expects to systematically stabilize the miscible phase as $R_{g} / R$ and/or colloid volume fraction increase. While capturing the essential physics, quantitative predictions are not available due to present limitations of PRISM m-PY that include the following: (i) only spinodals (rather than binodals for fluid-fluid phase boundaries) have been computed due to the technical complexities introduced by the thermodynamic self-consistency, (ii) only athermal polymers in good solvents have been studied (using the technical approximation of Gaussian coil statistics), and (iii) the theory cannot handle extremely strong depletion effects due to the use of the PY approximation for colloid-colloid correlations. In practice, the latter limitation is understood ${ }^{15,20}$ to be only important at low colloid volume fractions and $R \gg R_{g}$. It arises due to the linearized nature of the PY closure for colloid-colloid direct correlations. Such an approximation is also inherent to the classical models and other recently proposed theories such as the density functional approach. ${ }^{37}$ Indeed, the density functional approach reduces to the theory of Lekkerkerker et al. ${ }^{12}$ for the mixture free energy and phase behavior. Limitations (i) and (ii) can be relaxed by numerically intensive procedures as will be described in future work.

PRISM/m-PY spinodals for the experimental systems are shown in Fig. 8(a) as solid lines. The rapid divergence of the spinodal curves at small colloid volume fraction arises from the competition between increasing depletion strength but decreasing range as $c_{p}>c_{p}^{*}$ in good solvents, although limitation (iii) may also be relevant when $R>R_{g}$. Away from low $\phi, c_{p} / c_{p}^{*}$ values defining the spinodal monotonically increase with $R_{g} / R$ in accord with experiment. For comparison, we also show spinodals predicted by the Lekkerkerker et al. ${ }^{12}$ model as dashed lines. Comparisons of the PRISM spinodals with the measured binodals are shown in Fig. 8(b) for the three largest polymer samples, which display fluidfluid phase separation. The level of agreement is encouraging considering there are no adjustable parameters. Since the theory curves are spinodals and not binodals, quantitative comparisons are not appropriate, but better agreement does
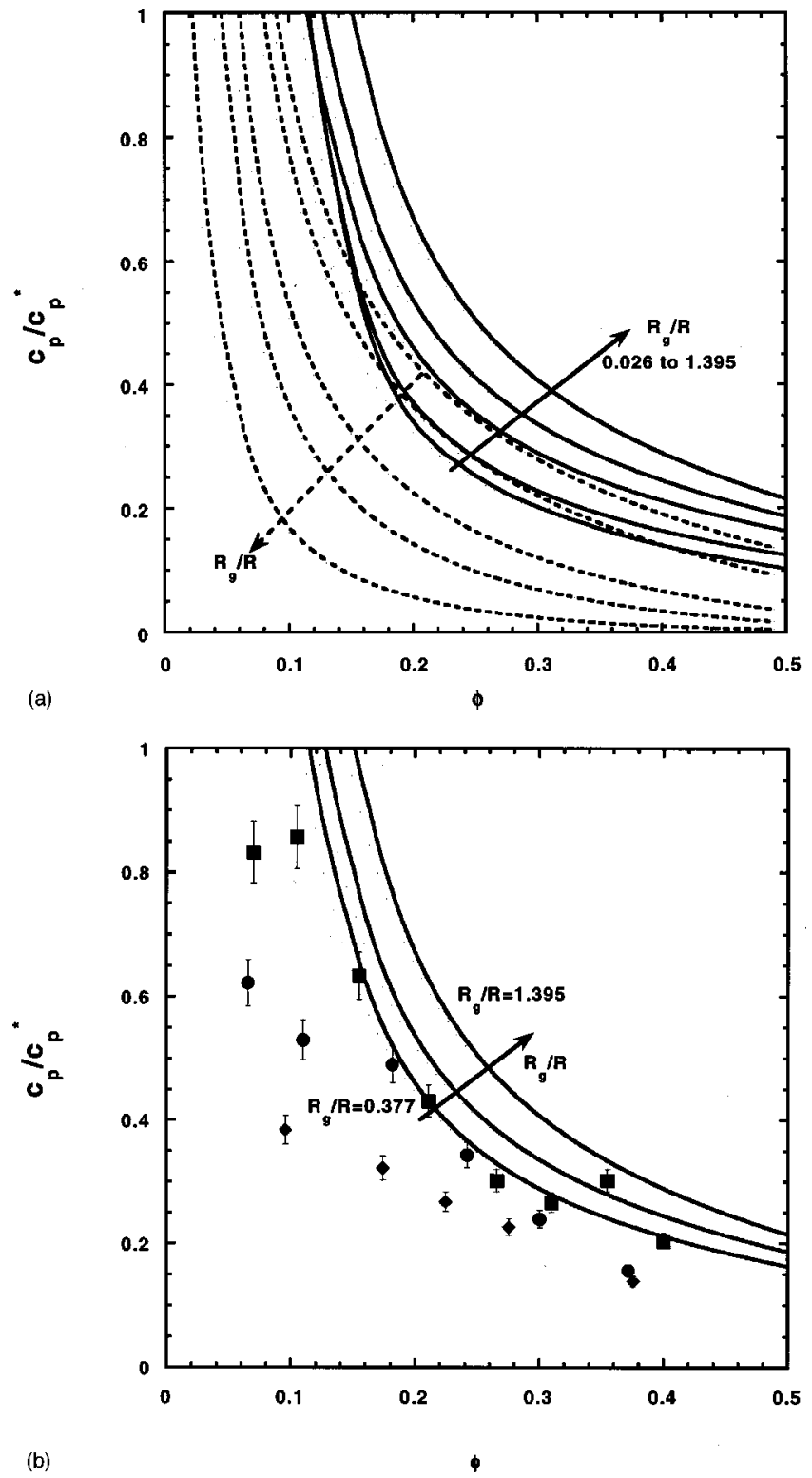

FIG. 8. (a) Fluid-fluid spinodals calculated from the PRISM m-PY theory (solid curves) and Lekkerkerker et al. theory (dashed curves) for the $R_{g} / R$ values of the experiments: $R_{g} / R=0.026,0.115,0.377,0.667$, and 1.395. (b) Comparisons of predicted spinodals of PRISM m-PY theory (solid curves) with the measured binodals for the three largest polymer samples. The solid symbols are the experimental points for $R_{g} / R=0.377$ (diamonds), 0.667 (circles), and 1.395 (squares).

seem to occur as $\phi$ increases, as expected based on limitation (iii) above. The spinodals predicted by the Lekkerkerker et al. ${ }^{12}$ theory shift in the opposite direction compared to PRISM and experiment. Their dependence on $R_{g} / R$ gets stronger with increasing colloid volume fraction, versus the behavior of PRISM and experiment, which shows a weak, if any, dependence.

Although fragmentary, there are a few experiments in the literature that show enhanced fluid phase stabilization with increasing $R_{g} / R$ ratio. Surfactant spherical micelles plus nonadsorbing water soluble polymers were examined by varying $R$ for relatively low micelle volume fractions; a range $R_{g} / R \sim 3-10$ was explored. ${ }^{38}$ Suspensions of small proteins plus free polymer have been also been studied. ${ }^{39}$ For 


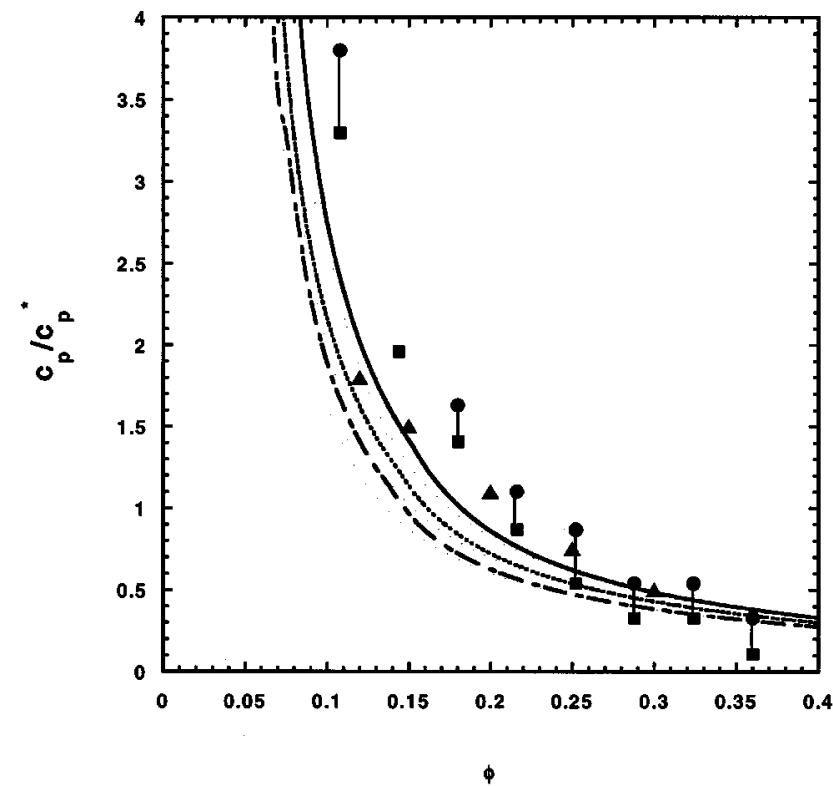

FIG. 9. Phase diagram for the whey protein-polymer mixture (Ref. 40). The fluid-fluid coexistence curve lies between the connected circles (phase separated) and square (miscible) data points. The corresponding PRISM m-PY spinodal is the solid curve. Fluid-fluid binodal data for the silica-polymer mixture (Ref. 41) are the solid triangles. The short dashed and long-short dashed curves are the PRISM m-PY spinodals for $R_{g} / R=1.9$ and 1.1 , respectively, which cover the range of the experimentally estimated size asymmetry.

the micelle and protein systems, the relevant polymer concentrations can easily penetrate well into the semidilute regime. As a recent example, data of Tuinier, Dhont, and de Kruif $^{40}$ are given in Fig. 9 for a small whey protein mixed with an exocellular polysaccharide where $R_{g} / R=3.2$. The $\mathrm{PRISM} / \mathrm{m}-\mathrm{PY}$ spinodal is in reasonable proximity of the experimental binodal, although the theory curve appears to be systematically lower. The latter trend may be a consequence of the use of an effectively Gaussian single-chain structure factor in PRISM, which overestimates coil internal density and hence underpredicts the penetrability of small particles into real self-avoiding coils, thereby overestimating depletion attraction. Interestingly, the experimental binodal also appears to become increasingly steep as protein concentration is decreased.

Fluid-fluid binodal data for a suspension of a small sterically stabilized silica colloid $(R \cong 10 \mathrm{~nm})$ mixed with polydimethylsiloxane in cyclohexane ${ }^{41}$ where $R_{g} / R \sim 1.4$ is also shown in Fig. 9, and is in reasonable accord with the theoretical spinodal. The binodal appears at higher polymer concentrations than found in Fig. 2. This may reflect the real physical and chemical differences between the experimental systems. We note, for example, that underlying soft repulsions may act to stabilize the fluid phase.

The origin of the ability of PRISM/m-PY to predict increased miscibility with increasing $R_{g} / R$ arises from multiple physical factors. The theory includes polymer chain structure (and hence particle penetration of polymer coils), polymer-polymer repulsions and physical mesh formation, many-body (if $R_{g} / R$ is not too small) effective depletion interactions in a true two-component mixture, and colloid and polymer clustering (both local and on large scales). ${ }^{20}$ Disentangling the consequences of these multiple "correlation effects" is not generally possible. However, we can clearly conclude that polymer-polymer interactions and correlations, present at nonzero polymer concentrations, must play a central role since we find that employing the PRISM m-PY insertion free energy (Fig. 4) in the classical approaches does not lead to any qualitative changes of the classic predictions. A crucial aspect of the PRISM/m-PY theory is its self-consistent prediction of a "nonlocality length" $\lambda$, which describes the distance from the particle surface over which the polymer conformational entropy is reduced. This length is connected to a depletion attraction range and varies from the polymer size when $R_{g} \ll R$, to a length of order $R$ when $R_{g} \gg R$. In general, $\lambda$ also depends on polymer and colloid concentration due to screening effects that are essential to take into account in computing the fluid-fluid spinodal boundary. ${ }^{20}$

\section{Gelation}

One of our major experimental findings is the transition from a homogenous fluid to a homogenous gel in the onephase region for very large size asymmetry, $R_{g} / R=0.026$. Prior studies on relatively dilute colloids, proteins, and other particle suspensions mixed with free polymer have generally also found nonequilibrium gel transitions, but seemingly always in the metastable (two-phase) region of the phase diagram. ${ }^{4,7,13,42}$ For example, for $R_{g} / R \sim 0.06-0.08$, Poon and co-workers ${ }^{4,7,13}$ found gel lines for colloid-polymer mixtures just barely above the fluid-crystal boundary. As $R_{g} / R$ increases, experiments appear to show the gelation phenomena moves deeper into the metastable two-phase region. ${ }^{42}$ Consistent with this, we also do not find gels as the first boundary encountered upon adding polymer when $R_{g} / R \geqslant 0.115$.

These observations raise the interesting scientific, and practically important, question of the relative sensitivity of equilibrium and nonequilibrium (gel) transitions to the polymer-colloid size asymmetry or the spatial range of the depletion attraction. Recently, Bergenholtz and co-workers ${ }^{23}$ have proposed a dynamic self-consistent mode coupling theory (MCT) to treat physical gelation of spherical particles interacting via a hard-core repulsion plus short-range squarewell attraction. An effective one-component fluid model is adopted. For $\Delta \sigma \ll 1$, and low colloid volume fractions, a simple analytic condition for gelation has been derived:

$$
\frac{12}{\pi^{2}} \frac{\Delta}{\sigma} \phi\left(e^{\beta \epsilon}-1\right)^{2}=1.42 .
$$

Full numerical solution $^{23}$ of the MCT equations are in good agreement with the analytic result if $\Delta / \sigma \ll 1$ and $\phi_{c}<0.4$. The mapping of the square-well parameters onto $R_{g} / R$ and $c_{p} / c_{p}^{*}$ can be done as described in Sec. IV B via Eqs. (14) and (15), with the result

$$
\left(\frac{c_{p}}{c_{p}^{*}}\right)_{\mathrm{gel}}=\alpha \frac{2}{3} \frac{R_{g}}{R} \ln \left[1+\left(\frac{3}{8} \frac{R}{R_{g}} \frac{1.42 \pi^{2}}{\phi}\right)^{1 / 2}\right] .
$$




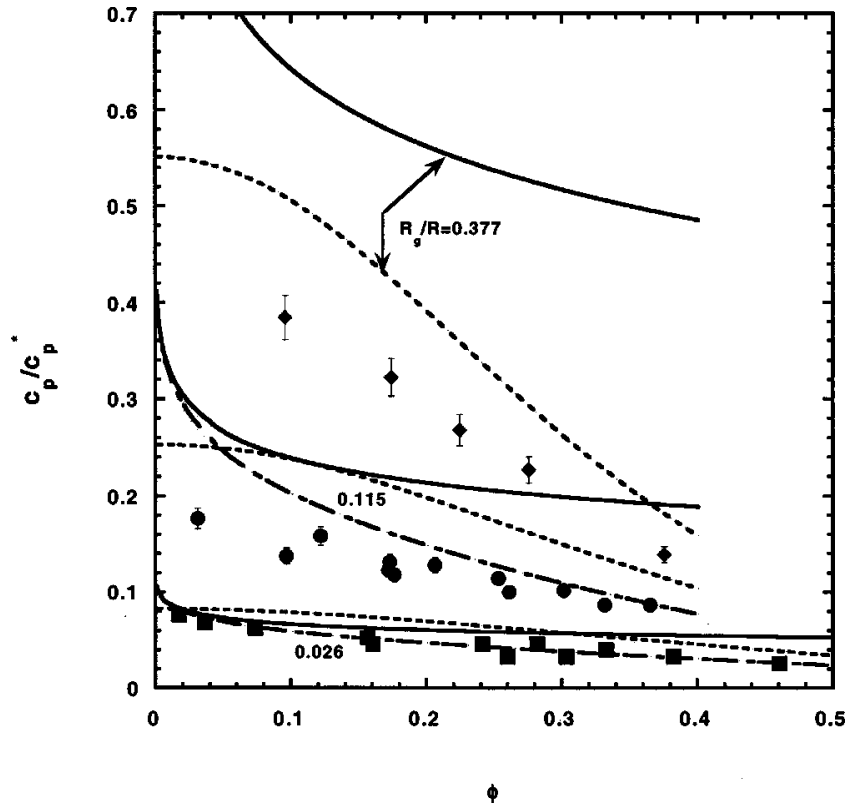

FIG. 10. MCT gel lines and static percolation lines compared with the experimental data. The solid symbols are the experimental points delimiting the stable fluid region for $R_{g} / R=0.026$ (squares), 0.115 (circles), and 0.377 (diamonds). The long-short dashed curves and the solid curves are MCT gel lines with (-- - ) and without (-) taking into account the polymer free-volume correction to $c_{p} / c_{p}^{*}$. The short dashed curves are the static percolation lines from the AHS model without the free-volume correction. For $R_{g} / R=0.377$, the long-short dashed curve dips well below the experimental data at higher $\phi$ and is not shown for clarity.

MCT gel lines for our experimental systems are shown in Fig. 10. Two versions have been given, corresponding to including, or not including, the polymer free-volume correction $\left(\alpha_{\mathrm{m}-\mathrm{PY}}\right)$ to $c_{p} / c_{p}^{*}$. In equilibrium, this correction factor is required, but the predictions without it are also shown for comparison since gelation is a nonequilibrium process, and Eq. (18) follows from a $\phi \ll 1$ analysis where $\alpha \rightarrow 1$. Remarkably, for the $R_{g} / R=0.026$ sample the MCT prediction is in near-quantitative agreement with the observed gel line. Although this level of agreement is presumably fortuitous, we do find it encouraging; note that for such small polymers the free-volume correction makes little difference. For $R_{g} / R$ $=0.115$, the predicted gel line lies in the metastable region of the experimental phase diagram, but is quite close to the fluid-crystal boundary. The latter fact seems consistent with prior studies ${ }^{7,13}$ on polymer-colloid mixtures with $R_{g} / R$ $\sim 0.1$. For $R_{g} / R=0.377$, the gelation line without the freevolume correction is again in the two-phase region, but it does drop sharply well below the equilibrium phase boundary at high $\phi$ if the free-volume correction is made (not shown for clarity). However, application of the effective onecomponent square-well model when $R_{g} / R$ is not so small is questionable.

Also shown in Fig. 10 are the static percolation lines computed using the PY solution of the AHS model, which are given by ${ }^{43}$

$$
\tau_{\text {percolation }}=\frac{19 \phi^{2}-2 \phi+1}{12(1-\phi)^{2}},
$$

where $\tau$ is the Baxter "stickiness" parameter. The same mapping as in Eqs. (11)-(15) is employed to make contact with the polymer-colloid mixture parameters, with the explicit result

$$
\left(\frac{c_{p}}{c_{p}^{*}}\right)_{\text {percolation }}=\alpha \frac{2}{3} \frac{R_{g}}{R} \ln \left[1+\frac{3 R}{R_{g}} \frac{(1-\phi)^{2}}{\left(19 \phi^{2}-2 \phi+1\right)}\right] .
$$

For clarity, the percolation lines are presented without the free-volume correction. Applying the correction (Fig. 4) lowers the curves in precisely the same manner as for the shown gel line examples. Interestingly, the percolation line and the dynamical MCT gel lines are close for small $R_{g} / R$. However, they have a qualitatively different shape, and the prediction of percolation at a finite $c_{p} / c_{p}^{*}$ as $\phi \rightarrow 0$ is an unphysical aspect of the theory of Ref. 43. In general, static percolation occurs increasingly earlier (lower $c_{p} / c_{p}^{*}$ ) relative to the dynamic MCT gel line as $R_{g} / R$ or $\phi$ increases. This behavior becomes more exaggerated if the free-volume correction is included (not shown). Even for $R_{g} / R=0.115$, the percolation line then falls below the equilibrium phase boundary at higher $\phi$ in contrast to experiment where no gels are seen before fluid-crystal phase separation. The trend that static percolation overestimates the ease of gel formation has been previously observed in sticky colloidal suspensions. Typically the percolation line is close to the observed gel line at low $\phi$ (at or below the critical volume fraction), but increasingly overestimates the gel line as the suspension is concentrated. ${ }^{44}$ Finally, we do believe the free-volume correction is likely appropriate and consistent to include for both the MCT and percolation approaches to gelation.

\section{SUMMARY AND CONCLUSIONS}

We have experimentally studied the phase behavior of a model athermal polymer-colloid suspension over a wide range of size asymmetry, colloid volume fraction, and polymer concentration. Our primary equilibrium finding is that based on $c_{p} / c_{p}^{*}$ as a dimensionless measure of polymerinduced depletion attractions, the suspension miscibility monotonically improves as the size asymmetry ratio $R_{g} / R$ increases. This conclusion applies regardless of whether the initial phase transition is fluid-crystal or fluid-fluid demixing, or even nonequilibrium gelation. Reduction of the polymer solution solvent quality results in a reduction of the value of $c_{p} / c_{p}^{*}$ required for phase separation but does not change the shifting up of fluid-fluid phase boundaries with increasing $R_{g} / R .^{13}$

The above experimental findings disagree with the predictions of all prior classic theories of which we are aware. The inadequacy of the latter must be tied to their inherent simplifications of polymer structure and/or suspension correlations that can include the following: (i) adoption of an effective one-component fluid model based on effective pair depletion potentials, (ii) treatment of polymers as hard spheres for describing their interaction with colloids, (iii) neglect of polymer-polymer correlations and interactions (effectively a $c_{p} / c_{p}^{*} \rightarrow 0$ assumption), and (iv) a thermodynamic perturbative treatment of the consequences of 
polymer-induced depletion attractions. Attempts to relax the latter approximation by using a more realistic onecomponent attractive sphere theory (AHS), or square-well fluid simulation data, also displayed qualitatively incorrect trends for the phase boundaries.

The microscopic two-component PRISM/m-PY theory ${ }^{20}$ predictions for fluid-fluid spinodal phase separation are in agreement with all the experimental trends. The critical role of nonzero polymer concentration and thermodynamic stateand $R_{g} / R$-ratio-dependent corrections to polymer-mediated depletion interactions can be deduced. Our recent systematic study of the colloidal osmotic compressibility of the present samples via light-scattering turbidity measurements reinforce these conclusions, ${ }^{45}$ as does prior quantitative, no-adjustableparameter comparisons of PRISM theory with small- and wide-angle scattering experiments on athermal colloidpolymer suspensions. $^{20,22}$ Wide-angle x-ray scattering measurements and exploration of the metastable phase behavior for our present systems are worthy future directions that can further test the integral equation theory. In addition, a systematic experimental study of the phase behavior in the "nanoparticle" regime, corresponding to $R_{g} \gg R$ and $c_{p}$ $\gg c_{p}^{*}$, will be very valuable. Improvement of several of the limitations of PRISM/m-PY theory discussed in Sec. IV C are needed to attain a more general and truly quantitative description.

Finally, we believe our discovery of a fluid-gel transition in the homogeneous one-phase region for the $R_{g}$ $=0.026 R$ sample is significant. The simple version of MCT proposed by Bergenholtz and co-workers ${ }^{23}$ appears to predict the location of the gel line remarkably well. Static percolation is also quite accurate for this very-short-range depletion attraction case. However, the latter seems to become increasingly inaccurate as $R_{g} / R$ (or $\phi$ ) increases, with percolation lines occurring in the one-phase homogeneous fluid regime, which appear to be irrelevant for the dynamic gelation phenomenon. On the other hand, the MCT predictions of gel lines for larger (but still small $R_{g} / R$ ) polymers appear qualitatively reasonable in the sense of being in the metastable regime as observed experimentally. A detailed study of our present silica system in the metastable regime to examine gel formation, structure, and dynamics is an interesting future direction.

\section{ACKNOWLEDGMENTS}

The work in Illinois was supported by the U.S. Department of Energy Division of Materials Science Grant No. DEFG02-96ER45539 through the Frederick Seitz Materials Laboratory. M.F. was supported by the Deutsche Forschungsgemeinschaft under Grant No. Fu 309/3 and through the SFB 563.

\footnotetext{
${ }^{1}$ For example, see chapters in Additives for Water Based Coatings, Proceedings of the Royal Society of Chemistry, Special Edition No. 165, edited by D. R. Karsa and W. D. Davies (The Royal Society of Chemistry, 1995).

${ }^{2}$ D. H. Napper, Polymeric Stabilization of Colloidal Dispersions (Academic, New York, 1983).

${ }^{3}$ B. Vincent, Adv. Colloid Interface Sci. 4, 193 (1974); P. R. Sperry, J. Colloid Interface Sci. 99, 97 (1984).
}

${ }^{4}$ W. C. K. Poon, A. D. Pirie, and P. N. Pusey, Faraday Discuss. 101, 65 (1995).

${ }^{5}$ J. Mewis, W. J. Frith, T. A. Strivens, and W. B. Russel, AIChE J. 35, 415 (1989).

${ }^{6}$ P. D. Patel and W. B. Russel, J. Rheol. 24, 287 (1980).

${ }^{7}$ P. N. Pusey, A. D. Pirie, and W. C. K. Poon, Physica A 201, 322 (1993).

${ }^{8}$ H. de Hek and A. Vrij, J. Colloid Interface Sci. 88, 258 (1982).

${ }^{9}$ C. Smits, B. van der Most, J. K. G. Dhont, and H. N. W. Lekkerkerker, Adv. Colloid Interface Sci. 42, 33 (1992).

${ }^{10}$ S. Asakura and F. Oosawa, J. Polym. Sci. 33, 183 (1958).

${ }^{11}$ A. P. Gast, C. K. Hall, and W. B. Russel, J. Colloid Interface Sci. 96, 251 (1983a,b); 109, 161 (1986).

${ }^{12}$ H. N. W. Lekkerkerker, W. C. K. Poon, P. N. Pusey, A. Stroobants, and P. B. Warren, Europhys. Lett. 20, 559 (1992).

${ }^{13}$ S. M. Ilett, A. Orrock, W. C. K. Poon, and P. N. Pusey, Phys. Rev. E 51, 1344 (1995).

${ }^{14}$ A. P. Chatterjee and K. S. Schweizer, Macromolecules 32, 923 (1999).

${ }^{15}$ A. P. Chatterjee and K. S. Schweizer, J. Chem. Phys. 109, 10464 (1998).

${ }^{16}$ A. P. Chatterjee and K. S. Schweizer, J. Chem. Phys. 109, 10477 (1998).

${ }^{17}$ K. S. Schweizer and J. G. Curro, Adv. Chem. Phys. 98, 1 (1997).

${ }^{18}$ A. M. Kulkarni, A. P. Chatterjee, K. S. Schweizer, and C. F. Zukoski, Phys. Rev. Lett. 83, 4554 (1999).

${ }^{19}$ A. M. Kulkarni, A. P. Chatterjee, K. S. Schweizer, and C. F. Zukoski, J. Chem. Phys. 113, 9863 (2000).

${ }^{20}$ (a) M. Fuchs and K. S. Schweizer, Europhys. Lett. 51, 621 (2000); (b) M. Fuchs and K. S. Schweizer, Phys. Rev. E 64, 021514 (2001).

${ }^{21}$ P. G. de Gennes, Scaling Concepts in Polymer Physics (Cornell University Press, Ithaca, NY, 1979).

${ }^{22}$ A. Moussaid, W. C. K. Poon, P. N. Pusey, and M. F. Soliva, Phys. Rev. Lett. 82, 225 (1999).

${ }^{23}$ J. Bergenholtz, M. Fuchs, and Th. Voigtmann, J. Phys.: Condens. Matter 12, 6575 (2000); J. Bergenholtz and M. Fuchs, Phys. Rev. E 59, 5706 (1999).

${ }^{24}$ W. Stober, A. Fink, and E. Bohn, J. Colloid Interface Sci. 26, 62 (1968).

${ }^{25}$ G. H. Bogush, G. H. Tracy, and C. F. Zukoski, J. Non-Cryst. Solids 114, 95 (1988).

${ }^{26}$ A. K. van Helden, J. W. Jansen, and A. Vrij, J. Colloid Interface Sci. 81, 354 (1981).

${ }^{27}$ K. D. Horner, M. Topper, and M. Ballauff, Langmuir 13, 551 (1997).

${ }^{28}$ W. B. Russel, D. A. Saville, and W. R. Schowalter, Colloidal Dispersions (Cambridge University Press, Cambridge, U.K., 1989).

${ }^{29}$ G. C. Berry, J. Chem. Phys. 44, 4550 (1966).

${ }^{30}$ B. Widom, J. Chem. Phys. 39, 2808 (1963).

${ }^{31}$ K. R. Hall, J. Chem. Phys. 57, 2252 (1972).

${ }^{32}$ R. J. Baxter, J. Chem. Phys. 49, 2770 (1968).

${ }^{33}$ R. O. Watts, D. Henderson, and R. J. Baxter, Adv. Chem. Phys. 21, 422 (1971).

${ }^{34}$ S. Ramakrishnan and C. F. Zukoski, J. Chem. Phys. 113, 1237 (2000).

${ }^{35}$ D. M. Heyes and P. J. Aston, J. Chem. Phys. 97, 5738 (1992).

${ }^{36}$ N. Asherie, N. Lomakin, and G. B. Benedek, Phys. Rev. Lett. 77, 4832 (1996).

${ }^{37}$ M. Dijkstra, J. Brader, and R. Evans, J. Phys.: Condens. Matter 11, 10079 (1999); M. Schmidt, H. Lowen, J. M. Brader, and R. Evans, Phys. Rev. Lett. 85, 1934 (2000).

${ }^{38}$ I. D. Robb, P. Williams, P. Warren, and R. Tanaka, J. Chem. Soc., Faraday Trans. 91, 3901 (1995); S. M. Clegg, P. Williams, P. Warren, and I. D. Robb, Langmuir 10, 3390 (1994).

${ }^{39}$ H. M. Schaink and J. A. Smit, J. Chem. Phys. 107, 1004 (1997).

${ }^{40}$ R. Tuinier, J. K. Dhont, and C. de Kruif, Langmuir 16, 1497 (2000).

${ }^{41}$ N. Verhaegh, J. van Duijneveldt, J. Dhont, and H. Lekkerkerker, Physica A 230, 409 (1996).

${ }^{42}$ M. Muschol and F. Rosenberger, J. Chem. Phys. 107, 1953 (1997).

${ }^{43}$ Y. C. Chiew and E. Glandt, J. Phys. A 16, 2599 (1983).

${ }^{44}$ M. Grant and W. B. Russel, Phys. Rev. E 47, 2606 (1993); H. Verduin and J. Dhont, J. Colloid Interface Sci. 172, 425 (1995).

${ }^{45}$ S. Ramakrishnan, M. Fuchs, K. S. Schweizer, and C. F. Zukoski, Langmuir (unpublished). 